${ }^{1}$ Клиника радиохирургии, стереотаксической радиотерапии и общей онкологии МИБС (Санкт-Петербург, Россия) Нейрохирургический институт им. проб. А.Л. Поленова, филиал «СЗФМИЦ» ил. В.А. Алмазова Минздрава России (Санкт-Петербург, Россия)

${ }^{3}$ Военно-медицинская акаделия им. С.М. Кирова, кафедра нейрохирургии (Санкт-Петербург, Россия)

${ }^{4}$ Санкт-Петербургский Государственный Университет (Санкт-Петербург, Россия)

5 ГБОУ ВПО СЗГМУ им. И.И. Мечникова (Санкт-Петербург, Россия)
РАДИОХИРУРГИЧЕСКОЕ ЛЕЧЕНИЕ МЕТАСТАЗОВ ЗЛОКАЧЕСТВЕННЫХ ОПУХОЛЕЙ ЖЕЛУДОЧНО-КИШЕЧНОГО ТРАКТА В ГОЛОВНОМ МОЗГЕ

П.И. Иванов ${ }^{1,2,3}$, И.С. Зубаткина ${ }^{1}$, В.В. Краснюк ${ }^{1}$ А.В. Кузьмин ${ }^{1}$, Н.А. Воробьев ${ }^{1,4,5}$

\section{RADIOSURGICAL TREATMENT OF BRAIN METASTASES FROM GASTROINTESTINAL CANCER}

П.И. Иванов ${ }^{1,2,3}$

Кандидат медицинских наук, нейрохирург, радиолог, заведующий отделением нейрорадиологии МИБС, ведущий научный сотрудник отделения нейроонкологии, ассистент кафедры нейрохирургии ВМА им. С.М. Кирова. Россия, Санкт-Петербург, 6-я Советская ул., 24 Тел.: 8 (921) 400-41-03, E-mail:ivanov@ldc.ru.

И.С. Зубаткина ${ }^{1}$ Кандидат биологических наук, медицинский физик отделения нейрорадиологии МИБС.

В.В. Краснюк ${ }^{1}$ Нейрохирург, отделение нейрорадиологии МИБС.

A.В. Кузимин Нейрохирург, отделение нейрорадиологии МИБС.

Н.A. Воробъев ${ }^{1,4,5}$ Кандидат медицинских наук, онколог, радиолог, заведующий отделением радиотерапии МИБС, доцент кафедры онкологии СПбГу; ассистент кафедры онкологии СЗГМУ им. И.И. Мечникова, Россия, Санкт-Петербург, ул. Кирочная, 41.

P.I. Ivanov ${ }^{1,2,3}$ Candidate of Medicine, Neurosurgeon, Radiologist, Head of Department of Neuroradiology, Radiosurgery, Stereotactic Radiotherapy and General Oncology clinic MIBS; Senior researcher of Department of Neurooncology, Assistant of the Department of Neurosurgery, Kirov Military Medical Academy; Polenov Russian Scientific Research Institute of Neurosurgery, Russia, Saint Petersburg, 6th Sovetskaya str., 24. Phone: 8 (921) 400-41-03, E-mail:ivanov@ldc.ru.

I.S. Zubatkina ${ }^{1}$ Candidate of Biological Sciences, Medical physicist of Department of Neuroradiology of MIBS.

V.V. Krasnyuk Neurosurgeon, Department of Neuroradiology of MIBS 


\section{A.V. Kuzmin ${ }^{1}$ \\ Neurosurgeon, \\ Department of Neuroradiology of MIBS.

N.A. Vorobyovi,4,5
Candidate of Medicine,
Oncologist, Radiologist,
Head of Department of Radiotherapy MIBS,
Associate Professor of Oncology Department,
St. Petersburg State University;
Assistant of the Department of Oncology,
Mechnikov North-West State Medical University,
Russia, Saint Petersburg, Kirochnaya str., 41.

Цель исследования. Проанализировать результаты радиохирургического лечения на аппарате Гамма-Нож пациентов с метастазами злокачественных опухолей ЖКТ в головном мозге с учетом прогностических факторов.

Материал и методы. В исследование включены 96 пациентов с метастазами рака ЖКТ в головном мозге, которым было проведено радиохирургическое лечение на Гамма-Ноже (Leksell Gamma Knife) в период с 2009 по 2016 годы. Продолжительность жизни, локальный контроль и появление новых метастазов в головном мозге изучались методом Каплана-Майера на основании катамнестических данных и контрольных МР-исследований.

Результаты. Медиана продолжительности жизни пациентов с метастазами рака ЖКТ в головном мозге после радиохирургического лечения на Гамма-Ноже составила 8,0 месяцев и статистически значимо различалась в зависимости От RPA класса: RPA III - 4,5 месяца, RPA II - 9,7 месяцев и RPA I - 18,5 месяцев. Локальный контроль (стабилизация или уменьшение размеров метастатических очагов) был достигнут в 94\% случаев. Появление новых метастазов в головном мозге отмечалось у $47 \%$ пациентов, медиана времени до выявления новых метастатических очагов в головном мозге составила 5,8 месяцев. Благоприятными факторами прогноза были отсутствие экстракраниальных метастазов и одиночный метастатический очаг в головном мозге.

Выводы. Радиохирургическое лечение позволяет достичь эффективного локального контроля метастазов злокачественных опухолей ЖКТ в головном мозге и существенно продлить жизнь пациентам, особенно в прогностически благоприятных классах.

Ключевые слова: метастазы в головноммозге, ракжелудочно-кишечного тракта, Гамиа-Нож, прогностические факторы, RРА-классы.

Objectives. The study aims to analyze the results of Gamma Knife radiosurgery for patients with brain metastases from gastrointestinal cancer taking into consideration prognostic factors.

Material and methods. The study includes 96 patients with GI metastases in the brain, who underwent radiosurgical treatment with Leksell Gamma Knife from 2009 to 2016. Overall survival, local control and the appearance of new metastases in the brain were studied by the Kaplan-Meier method based on follow-up data and control MRI.

Results. Median overall survival after Gamma Knife radiosurgery was 8,0 months for the entire cohort and differed significantly depending on the RPA class: RPA III - 4,5 months, RPA II - 9,7 months and RPA I - 18,5 months. Local control (stabilization or reduction in tumor volume) was achieved in $94 \%$ of cases. The appearance of new metastases in the brain was observed in $47 \%$ of patients; the median time to detect new metastatic lesions was 5,8 months. Favorable prognostic factors were a solitary metastasis in the brain and the absence of extra cranial disease.

Conclusion. Radiosurgical treatment allows one to achieve effective local control of gastrointestinal metastases in the brain and significantly prolong the patients' life, especially in prognostically favorable classes.

Keywords: brain metastases, gastrointestinal cancer, Gamma-Knife, prognostic factors, RPA classes.

\section{Введение}

3 локачественные опухоли ЖКТ достаточно редко прогрессируют с вовлечением центральной нервной системы: частота метастатического поражения головного мозга не превышает 4\% при раке пищевода, желудка, толстого кишечника и прямой кишки $[1,2]$. Метастазы рака ЖКТ в головном мозге в большинстве случаев возникают на поздней стадии онкологического заболевания и являются крайне неблагоприятным прогностическим фактором: ме- диана продолжительности жизни таких пациентов варьирует от 1 до 6 месяцев [3, 4]. В последние годы наблюдаются качественные изменения в системном лечении пациентов со злокачественными опухолями ЖКТ, связанные с внедрением таргетной терапии, направленной на молекулярную модуляцию опухолевых клеток [5]. Однако вследствие избирательной проницаемости гемато-энцефалического барьера действие современных лекарственных препаратов ограничивается в основном подавлением диссеминации опухоли за пределами ЦНС, что обуславливает 
повышение вероятности метастатического поражения головного мозга и вместе с тем необходимость определения эффективной тактики локального лечения, направленного на контроль распространенности заболевания в пределах ЦНС.

В настоящее время произошла смена парадигмы лечения метастазов рака в головном мозге: все в большем числе случаев отдается предпочтение высокоэффективному однократному прицельному облучению метастатических очагов высокими дозами радиации, вместо паллиативного облучения всего объема головного мозга [6]. Согласно клиническим рекомендациям Американского общества радиационных онкологов (ASTRO), радиохирургия рассматривается как приоритетный метод лечения одиночных или множественных метастазов в головном мозге, наибольший размер которых не превышает 3-4 см, в случае, если онкологический пациент имеет благоприятный прогноз (ожидаемая продолжительность жизни 3 и более месяцев) [7].
Целью данного исследования было проанализировать результаты практического применения радиохирургии для лечения метастазов рака ЖКТ в головном мозге на основе базы данных Радиохирургического центра МИБС и определить благоприятные факторы прогноза.

\section{Материал и методы}

\section{Дизайн исследования}

Одноцентровое когортное исследование, включающее 96 пациентов, которые проходили радиохирургическое лечение на аппарате Гамма-Нож с февраля 2009 по декабрь 2016 по поводу метастазов рака ЖКТ в головном мозге.

\section{Характеристика пащиентов}

Критериями отбора пациентов в исследование служили: гистологически верифицированный онкологический процесс; локализация первичной опухоли в ЖКТ; изолированные метастатические очаги в головном мозге; отсутствие клинических и

Таблища 1.

Характеристика пациентов с метастазами рака жкТ в головном мозге на момент поступления для радиохирургического лечения на аппарате Гамма-Нож (N=96 пациентов)

\begin{tabular}{|c|c|}
\hline Характеристика & Значение \\
\hline \multicolumn{2}{|l|}{ Возраст (годы) } \\
\hline Средний возраст & 61 \\
\hline Разброс & $34-79$ \\
\hline \multicolumn{2}{|l|}{ Пол } \\
\hline Мужчины & 46 \\
\hline Женщины & 50 \\
\hline \multicolumn{2}{|l|}{ Локализация первичной опухоли (количество пациентов) } \\
\hline Пищевод & 5 \\
\hline Желудок & 16 \\
\hline Толстый кишечник & 51 \\
\hline Прямая кишка & 24 \\
\hline \multicolumn{2}{|l|}{ Состояние первичного очага (количество пациентов) } \\
\hline Контроль & 92 \\
\hline Прогрессия & 4 \\
\hline \multicolumn{2}{|l|}{ Экстракраниальные метастазы } \\
\hline Выявлены & 68 \\
\hline Не выявлены & 28 \\
\hline \multicolumn{2}{|l|}{ Состояние по шкале Карновского (баллы) } \\
\hline Медиана & 70 \\
\hline Разброс & $50-90$ \\
\hline \multicolumn{2}{|l|}{ Неврологический статус (количество пациентов) } \\
\hline Общемозговая симптоматика & 90 \\
\hline Очаговая симптоматика & 42 \\
\hline \multicolumn{2}{|l|}{$\begin{array}{l}\text { Лечение метастазов в головном мозге до радиохирургии } \\
\text { (количество пациентов) }\end{array}$} \\
\hline Нейрохирургическая операция & 18 \\
\hline Облучение всего объема головного мозга & 4 \\
\hline \multicolumn{2}{|l|}{$\begin{array}{l}\text { Количество метастазов в головном мозге на момент радиохирургического лечения } \\
\text { (количество пациентов) }\end{array}$} \\
\hline Одиночный & 43 \\
\hline Множественные & 53 \\
\hline
\end{tabular}


рентгенологических данных за генерализованное поражение черепно-мозговых нервов и оболочек головного мозга; наибольший размер метастатического очага менее 3,5 см. Общая характеристика группы пациентов приведена в таблице 1.

Первичная опухоль была расположена в верхних отделах ЖКТ у 21 пациента (опухоль пищевода в 5 случаях, желудка в 16 случаях) и в нижних отделах ЖКТ у 75 пациентов (опухоль толстого кишечника в 51 случае, прямой кишки в 24 случаях). У одиннадцати пациентов был диагностирован первично-множественный рак ЖКТ. Гистологически доминировала аденокарцинома различной степени дифференцировки, плоскоклеточный рак был верифицирован при опухолевом поражении пищевода у 3 пациентов. У 83 (86\%) пациентов метастатическое поражение головного мозга было выявлено в результате постепенного прогрессирования онкологического заболевания в среднем через 26 месяцев с момента начала лечения первичной опухоли, у 13 (14\%) пациентов метастазы в головной мозг оказались первым проявлением онкологического процесса, и первичный очаг был обнаружен при дообследовании.

Экстракраниальные метастатические очаги на момент радиохирургического лечения были выявлены у 68 (71\%) пациентов. В большинстве случаев отмечался полиорганный характер опухолевой диссеминации. Метастазы в легких были выявлены у 46 пациентов, в печени - у 31, в костях - у 12. У 2 пациентов была поражена селезенка, у 1 - поджелудочная железа. Дополнительно отмечались метастатические очаги в мягких тканях малого таза, забрюшинном пространстве (таблица 2).

Из 96 пациентов 18 ранее были выполнены нейрохирургические вмешательства, удалено в общей сложности двадцать метастатических очагов. Четыре пациента прошли курс облучения всего объёма головного мозга. В данном случае, показанием к радиохиругическому лечению было появление новых очагов или продолженный рост очагов ранее подвергавшихся лечению.
При поступлении оценивался общесоматический и неврологический статус пациентов. Лечение проходили пациенты с общим состоянием по шкале Карновского от 50 баллов до 90 баллов (среднее значение 70 баллов). Всего у шести пациентов не наблюдалась общемозговая симптоматика, и поражение головного мозга было выявлено в процессе скринингового обследования. В сорока двух случаях отмечалась очаговая симптоматика, представленная гемипарезами различной степени выраженности, афотическими нарушениями и др. В двенадцати случаях метастазы в головном мозге провоцировали развитие эпилептических приступов.

Характеристика радиохирургического лечения на Гамла-Ноже

Радиохирургическое лечение проводилось на аппарате Leksell Gamma Knife 4C (Elekta AB, Sweden) до августа 2015 года и Leksell Gamma Knife Perfexion (Elekta AB, Sweden) с сентября 2015 года. Процедура и этапы лечения подробно описаны ранее [8, 9].

Всего проведено 134 процедуры радиохирургического лечения на аппарате Гамма-Нож, в 27 случаях 2 и более процедуры лечения требовались в связи с выявлением новых метастазов в головном мозге или необходимостью этапного лечения вследствие большого количества метастатических очагов. Радиохирургическое лечение одиночных метастазов в головной мозг проводилось 46 пациентам; 2-4 очагов - 32 пациентам; 5-10 - 11 пациентам; $>10$ - 7 пациентам. Всего было пролечено 307 метастатических опухолей. Средний объем наибольшего метастаза составил 8,5 см$^{3}$ (от 0,2 до 27,5 $\mathrm{cm}^{3}$ ). Средний суммарный объем всех метастатических очагов у одного пациента составил $10,9 \mathrm{~cm}^{3}$ (от 0,5 до 60,5 $\left.\mathrm{cm}^{3}\right)$. У 54\% пациентов метастазы были расположены в больших полушариях головного мозга, у 15\% - в задней черепной ямке. Дозы облучения по краю мишени выбирались с учетом рекомендаций RTOG, в большинстве случаев в диапазоне от 18 до 22 Гр (средняя доза 19,3 Гр), предписанная изодоза варьировала от 40 до 85\% (обычно 50\%).

Распространенность опухолевого процесса у пациентов с метастазами рака жкт в головном Таблица на момент радиохирургического лечения (N=96 пациентов).

\begin{tabular}{|l|c|c|c|c|}
\hline \multirow{2}{*}{\multicolumn{1}{|c|}{ Характеристика }} & \multicolumn{3}{|c|}{ Локализация первичной опухоли } \\
\cline { 2 - 5 } & $\begin{array}{c}\text { Пищевод } \\
\text { (5 пациентов) }\end{array}$ & $\begin{array}{c}\text { Желудок } \\
\text { (16 пациентов) }\end{array}$ & $\begin{array}{c}\text { Толстый } \\
\text { кишечник } \\
\text { (51 пациент) }\end{array}$ & $\begin{array}{c}\text { Прямая кишка } \\
\text { (24 пациента) }\end{array}$ \\
\hline $\begin{array}{l}\text { Метастазы только } \\
\text { в головном мозге }\end{array}$ & $2(40 \%)$ & $5(31 \%)$ & $7(29 \%)$ \\
\hline $\begin{array}{l}\text { Метастазы в головном } \\
\text { мозге и экстракраниальные } \\
\text { метастазы }\end{array}$ & $3(60 \%)$ & $11(69 \%)$ & $36(71 \%)$ & $17(71 \%)$ \\
\hline Метастазы в легких & $1(33 \%)$ & $4(36 \%)$ & $28(78 \%)$ & $13(76 \%)$ \\
\hline Метастазы в печени & $1(33 \%)$ & $2(18 \%)$ & $21(58 \%)$ & $7(41 \%)$ \\
\hline
\end{tabular}




\section{Статистические методы}

Статистический анализ материала проводился в программе SPSS Statistics 17.0 (USA). Продолжительность жизни пациентов, локальный контроль и появление новых метастазов в головном мозге изучались с помощью метода анализа цензурированных данных Каплана-Майера. Цензурирование осуществлялось на основании контрольных МРТ исследований головного мозга, информации о состоянии и продолжительности жизни, полученной в результате телефонного разговора с пациентами и их родственниками, а также по данным очных консультаций. Временные данные представлены в виде медианы и 95\% доверительного интервала (95\% ДИ), кумулятивные - в процентном отношении. Сравнение продолжительности жизни в группах проводилось с помощью непараметрического логарифмического рангового критерия. Статистически значимыми считались различия при величине $\mathrm{p}<0,05$.

\section{Результаты}

Продолжительность жизни

Медина наблюдения пациентов после радиохирургии составила 7 месяцев (от 1 до 78 месяцев). На момент статистического анализа материала (сентябрь 2017 года), 11 пациентов оставались живы. Медиана продолжительности жизни после радиохирургического лечения на Гамма-Ноже составила 8,0 месяцев (95\% ДИ 6,2 - 9,7 месяцев). Актуарная 6-месячная выживаемость - 67\%, однолетняя выживаемость - 35\%, двухлетняя выживаемость - 13\% (рис. 1А).

Продолжительность жизни пациентов после радиохирургии при локализации первичной опухоли в верхних отделах ЖКТ составила 9,7 месяцев (95\% ДИ), в нижних отделах ЖКТ 7,8 месяцев (95\% ДИ), однако статистически значимой разницы между группами достигнуто не было (p=0,758).

Факторами благоприятного прогноза оказались более молодой возраст пациента, высокий балл по шкале Карновского, отсутствие экстракраниальных метастазов и одиночный метастатический очаг в головном мозге. Локализация метастазов в головном мозге, объем наибольшего очага, суммарный объем всех очагов, контроль первичной опухоли, пол пациента статистически значимо не влияли на продолжительность жизни после радиохирургического лечения (таблица 3).

Таблица 3.

Однофакторный анализ продолжительности жизни пациентов с метастазами рака жкТ в головном мозге после радиохирургического лечения на Гамма-Ноже

\begin{tabular}{|c|c|c|c|}
\hline Параметр & $\begin{array}{c}\text { Количество } \\
\text { пациентов }\end{array}$ & $\begin{array}{c}\text { Медиана } \\
\text { выживаемости (месяцы) }\end{array}$ & $\begin{array}{l}\text { Достоверность } \\
\text { (Log Rank test) }\end{array}$ \\
\hline \multicolumn{4}{|l|}{ Возраст } \\
\hline$<60$ & 39 & 12,5 & $\mathrm{P}=\mathbf{0 , 0 0 9}$ \\
\hline$\geq 60$ & 57 & 7,8 & \\
\hline \multicolumn{4}{|l|}{ Пол } \\
\hline Женщины & 50 & 7,6 & $\mathrm{P}=0,723$ \\
\hline Мужчины & 46 & 8,8 & \\
\hline \multicolumn{4}{|l|}{ Статус по шкале Карновского } \\
\hline$\geq 70$ & 69 & 10,6 & $\mathbf{P}<0,0001$ \\
\hline$<70$ & 27 & 4,6 & \\
\hline \multicolumn{4}{|l|}{ Состояние первичного очага } \\
\hline Контроль & 92 & 8,0 & $\mathrm{P}=0,174$ \\
\hline Прогрессия & 4 & 1,9 & \\
\hline \multicolumn{4}{|l|}{ Экстракраниальные метастазы } \\
\hline Не выявлены & 28 & 15,1 & $\mathrm{P}=0,005$ \\
\hline Выявлены & 68 & 6,9 & \\
\hline \multicolumn{4}{|l|}{ RPA } \\
\hline RPA I & 17 & 18,5 & $\mathbf{P}<0,0001$ \\
\hline RPA II & 52 & 9,7 & \\
\hline RPA III & 27 & 4,6 & \\
\hline \multicolumn{4}{|l|}{ Количество метастазов в головном мозге } \\
\hline 1 & 46 & 11,1 & $\mathrm{P}=\mathbf{0 , 0 0 9}$ \\
\hline$>1$ & 50 & 6,4 & \\
\hline \multicolumn{4}{|l|}{ Локализация метастазов в головном мозге } \\
\hline Супратенториальные & 52 & 9,7 & $\mathrm{P}=0,091$ \\
\hline Инфратенториальные & 14 & 10,6 & \\
\hline Супра-инфратенториальные & 30 & 6,5 & \\
\hline \multicolumn{4}{|l|}{ Объем наибольшего метастаза } \\
\hline$<10 \mathrm{CM}^{3}$ & 66 & 8,6 & $\mathrm{P}=0,802$ \\
\hline$\geq 10 \mathrm{~cm}^{3}$ & 30 & 6,9 & \\
\hline
\end{tabular}



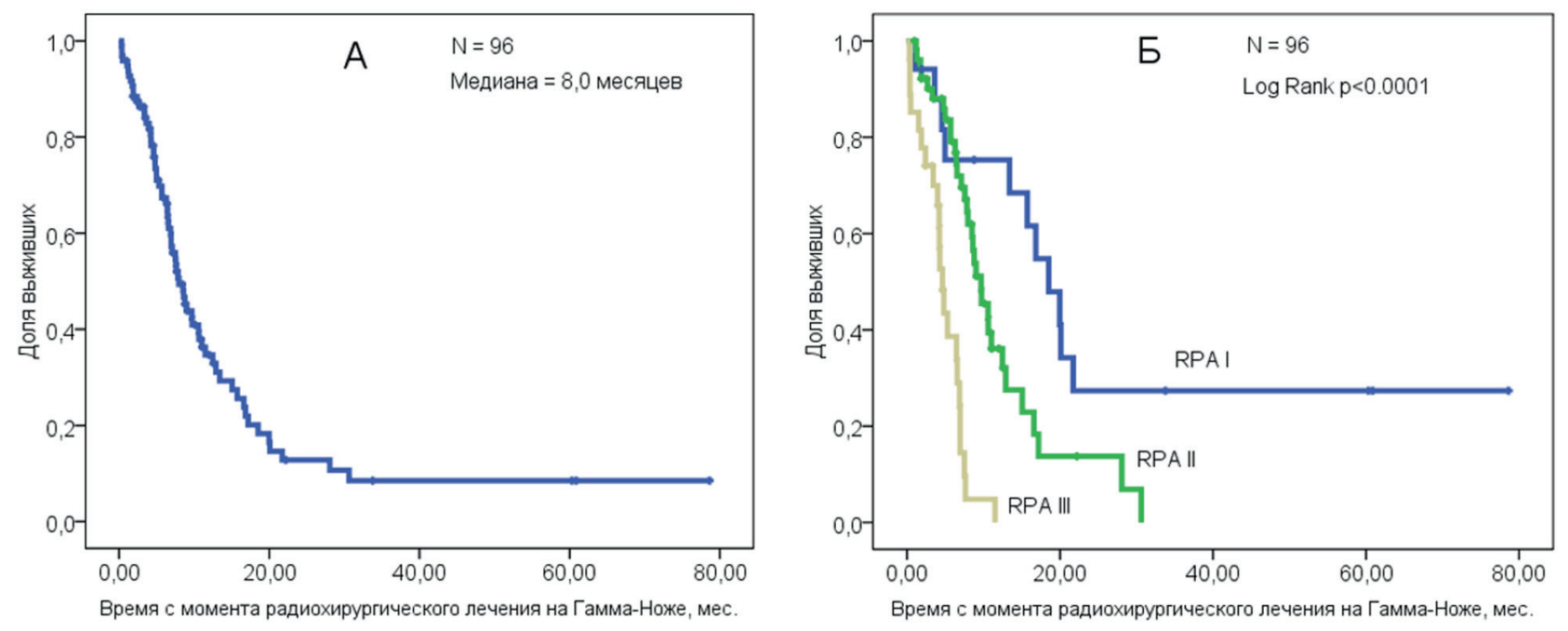

Pис. 1. Продолжительность жизни пащиентов сметастазами рака ЖКТ в головном мозге после радиохирургического лечения на аппарате Гамла-Нож: А - для группы в целом, Б - в зависимости от RPA класса.

Стратификация пациентов была проведена по наиболее распространенной прогностической системе, предложенной Gaspar et al. в 1996 году [10]. Данная система предполагает распределение пациентов по 3 прогностическим классам на основании 4 клинических параметров: возраста, состояния по шкале Карновского, контроля первичной опухоли и наличия экстракраниальных метастазов. В данном исследовании у 17 пациентов, относящихся к наиболее благоприятному первому классу RPA I, медиана продолжительности жизни составила 18,5 месяцев (95\% Ди 13,3 - 23,7 месяцев), однолетняя выживаемость $75 \%$, двухлетняя 27\%. У 27 пациентов, относящиеся к наименее благоприятному прогностическому классу RPA III, медиана продолжительности жизни после радиохирургии составила 4,5 месяца (95\% ДИ 3,8 5,5 месяцев). Однолетняя выживаемость в этой группе не была достигнута. Наибольшее количество

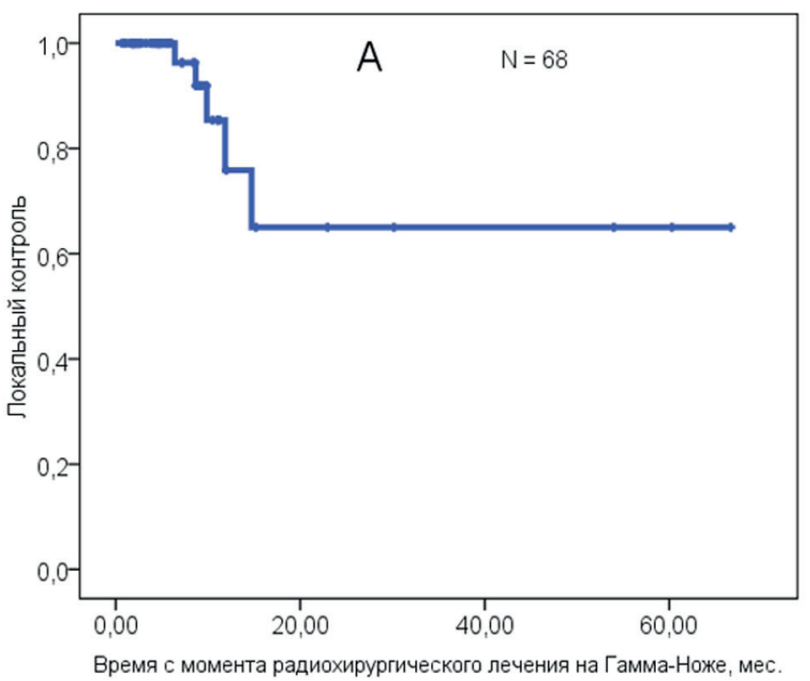

пациентов было отнесено ко второму прогностическому классу RPA II (52 человека), в котором медиана продолжительности жизни составила 9,7 месяцев (95\% ДИ 7,5 - 11,9 месяцев), однолетняя выживаемость 36\%, двухлетняя выживаемость 14\% (рис. 1Б).

\section{Локальный контроль}

Эффективность радиохирургического лечения и радиологический ответ опухоли изучались на основании контрольных МРТ исследований, которые пациенты регулярно выполняли с частотой в 2-3 месяца после лечения. Контрольные МРТ снимки импортировались в программу Leksell Gamma Plan FollowUр, где они совмещались со стереотаксическими МР-изображениями, полученными во время лечения. Контрольные МРТ исследования были доступны для анализа у 68 пациентов. Локальный контроль (уменьшение или стабилизация опухоли) был достигнут у 64 пациентов, в 4 случаях был выявлен продолженный

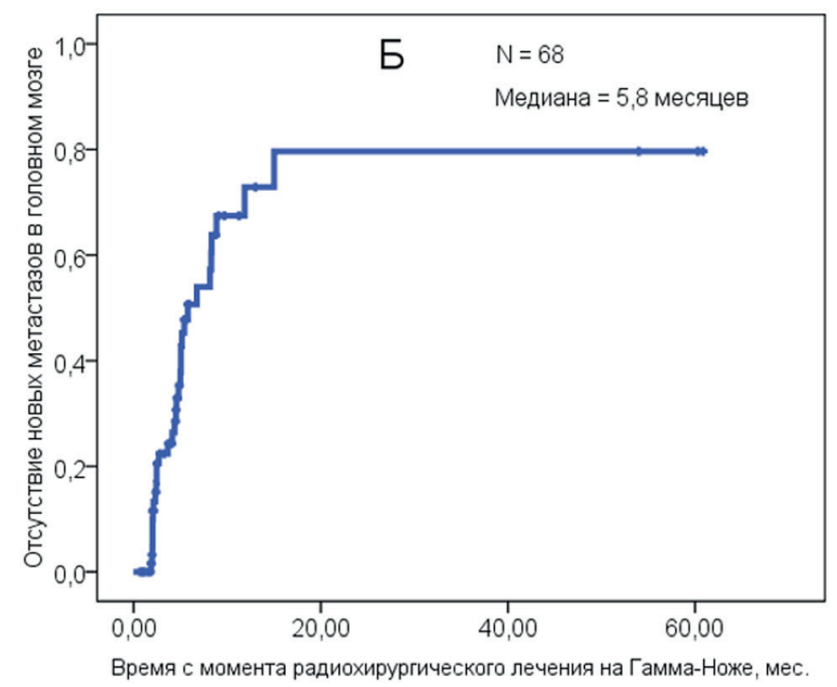

Рис. 2. Локальный контроль (А) и отсутствие новых метастазов в головном мозге (Б) у пациентов слетастазами рака ЖКТ в головном мозге после радиохирургического лечения на аппарате Гамиа-Нож. 


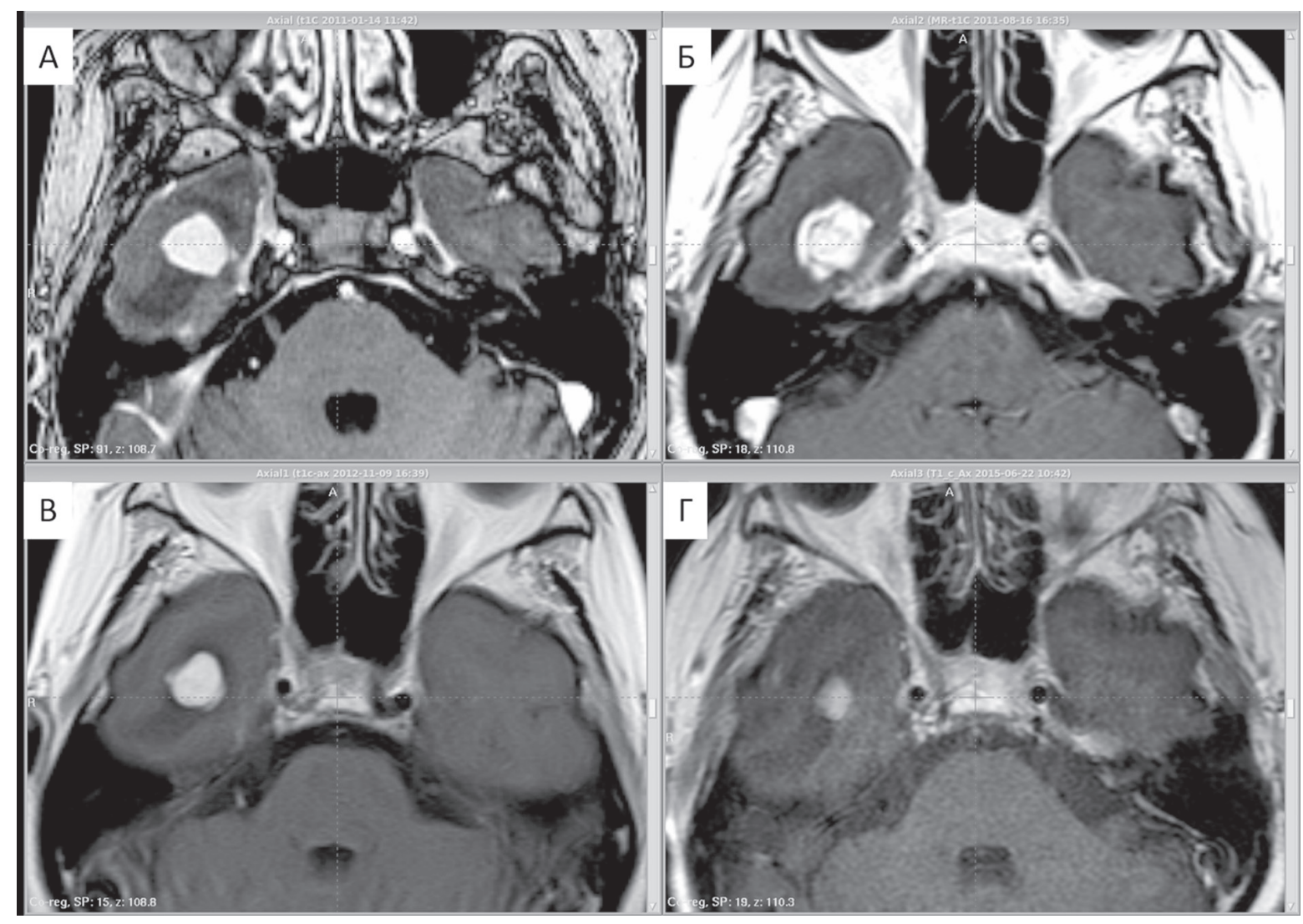

Pис. 3. Долгосрочный динамический МР-контроль метастаза рака ЖКТ в правой височной доле головного мозга после радиохирургического лечения на аппарате Гамма-Нож (А - Г Т1-взвешенные МР-изображения с контрастным усилением):

$A$ - на момент радиохирургии, Б - через 7 месячев увеличение размеров опухоли и перифокального отека вследствие постлучевых реакиий, B - через 22 месяча уменьшение размеров опухоли, $\Gamma$ - через 4,5 года дальнейшее уменьшение опухоли.

рост. Время до возникновения продолженного роста варьировало от 8,7 до 14,7 месяцев после лечения с медианой в 11,3 месяцев. 12-месячный уровень локального контроля составил 76,9\% (рис. 2А).

У 19 пациентов на протяжении периода наблюдения было выявлено временное увеличение размеров метастатической опухоли, связанное с постлучевыми реакциями, которые проявлялись временным увеличением зоны накопления контраста на постконтрастных Т1 изображениях и перифокального отека на Т2 изображениях локально в месте лучевого воздействия. Постлучевые реакции наиболее ярко проявлялись на МРТ через 6 месяцев после радиохирургии, затем выраженность изменений постепенно уменьшалась (рис. 3). При клиническом проявлении постлучевых реакций назначалась противоотечная терапия.

Появление новых метастазов в головном мозге

После радиохирургического лечения на контрольных МРТ исследованиях новые метастатические очаги в головном мозге были обнаружены у 32 (47\%) из 68 пациентов. Медиана времени до выявления новых метастазов в головном мозге с момента радиохирургического лечения составила 5,8 месяцев (95\% Ди 2,7 - 8,9 месяцев). Вероятность отсутствия новых очагов в головном мозге на протяжении первых 6 месяцев после радиохирургии оценивается в
49,3\%, 12 месяцев - в 27,1\%. В случае выявления новых метастазов пациентам проводилось повторное радиохирургическое лечение (рис. 2Б).

Статистически значимыми факторами риска появления новых метастазов в головном мозге оказались множественные (2 и более) метастатические очаги в головном мозге на момент поступления пациента на радиохирургическое лечение и наличие экстракраниальных метастазов (таблица 4).

\section{Обсуждение}

Метастатическое поражение головного мозга у пациентов со злокачественными опухолями ЖКТ встречается достаточно редко (4\%) и считается индикатором поздней стадии онкологического процесса, для которой свойственна широкая диссеминация первичной опухоли в организме $[2,11]$. У пациентов с метастатическим распространением рака ЖКТ в головной мозг в большинстве случаев уже имеются метастатические очаги в легких (63\%), печени (39\%), костях (5-26\%), лимфатических узлах (7-36\%) и забрюшинном пространстве (3-25\%). Отмечается зависимость между наличием метастазов в легких и последующим возникновением метастазов в головном мозге $[12,13]$. В данном исследовании метастатические очаги в головном мозге сочетались 
Однофакторный анализ отсутствия новых метастазов головном мозге

Таблица 4. после радиохирургического лечения на Гамма-Ноже ( $\mathrm{N}=68$ пациентов)

\begin{tabular}{|c|c|c|c|}
\hline Параметр & $\begin{array}{l}\text { Количество } \\
\text { пациентов }\end{array}$ & $\begin{array}{l}\text { Медиана, } \\
\text { месяцы }\end{array}$ & $\begin{array}{l}\text { Достоверность } \\
\text { (Log Rank test) }\end{array}$ \\
\hline \multicolumn{4}{|l|}{ Возраст } \\
\hline$<60$ & 31 & 8,3 & $\mathrm{P}=0,140$ \\
\hline$\geq 60$ & 37 & 5,0 & \\
\hline \multicolumn{4}{|l|}{ Пол } \\
\hline Женщины & 35 & 5,8 & $\mathrm{P}=0,888$ \\
\hline Мужчины & 33 & 5,2 & \\
\hline \multicolumn{4}{|l|}{ Статус по шкале Карновского } \\
\hline$\geq 70$ & 54 & 6,8 & $\mathrm{P}=0,281$ \\
\hline$<70$ & 14 & Среднее 4,1 & \\
\hline \multicolumn{4}{|l|}{ Состояние первичного очага } \\
\hline Контроль & 65 & 6,8 & $\mathrm{P}=0,225$ \\
\hline Прогрессия & 3 & 2,7 & \\
\hline \multicolumn{4}{|l|}{ Экстракраниальные метастазы } \\
\hline Не выявлены & 20 & Среднее 37,6 & $\mathrm{P}=\mathbf{0 , 0 1 0}$ \\
\hline Выявлены & 48 & 5,1 & \\
\hline \multicolumn{4}{|l|}{ Количество метастазов в головном мозге } \\
\hline 1 & 37 & 8,9 & $\mathrm{P}<0,0001$ \\
\hline$>1$ & 31 & 4,6 & \\
\hline \multicolumn{4}{|l|}{ Нейрохирургическая операция } \\
\hline Не проводилась & 52 & 6,8 & $\mathrm{P}=0,341$ \\
\hline Проводилась & 16 & 5,4 & \\
\hline \multicolumn{4}{|l|}{$\begin{array}{l}\text { Время выявление метастазов } \\
\text { в головном мозге }\end{array}$} \\
\hline $\begin{array}{l}\text { Одновременно с первичной } \\
\text { опухолью }\end{array}$ & 9 & 8,3 & $\mathrm{P}=0,785$ \\
\hline Последовательно & 59 & 5,4 & \\
\hline
\end{tabular}

с метастатическим поражением внутренних органов, костей и мягких тканей в $71 \%$ случаев, при этом преобладал полиорганный характер диссеминации. Метастазы в легких были выявлены у $78 \%$ и $76 \%$ пациентов с аденокарциномой толстой кишки и прямой кишки, соответственно. Пациенты с раком пищевода и желудка имели более низкую частоту одновременной встречаемости метастазов в головном мозге и в легких: 33\% и 36\%, соответственно. Сочетание метастатического поражения печени и головного мозга имело схожую зависимость от локализации первичной опухоли в ЖКТ: $58 \%$ и 41\% при опухолях толстого кишечника и прямой кишки, 18\% и 33\% при опухолях желудка и пищевода. Таким образом, данные настоящего исследования подтверждают ранее выявленные особенности сочетанного метастазирования злокачественных образований ЖКТ во внутренние органы и головной мозг, что отражает их генерализованный характер метастатического распространения. Данная особенность обуславливает крайне неблагоприятный прогноз этой группы пациентов при отсутствии эффективного комплексного лечения, направленного на контроль метастазов в головном мозге и системную терапию.
Внедрение радиохирургии в широкую клиническую практику и её подтвержденная эффективность в отношении контроля опухолевого роста качественно изменили возможности лечения метастазов рака в головном мозге. По данным клинических исследований, результаты радиохирургического лечения сопоставимы с результатами хирургического удаления с учетом стратификации пациентов по прогностическим шкалам. При этом хирургическое удаление предпочтительно при одиночных опухолях большого размера, расположенных в доступных для хирургического вмешательства областях головного мозга, и возможности проведения общей анестезии. Радиохирургия может быть применена для лечения опухолей любой локализации, в том числе расположенных в глубинных и функционально значимых структурах головного мозга, а также множественных метастатических очагов. Было показано, что радиохирургическое лечение имеет одинаковую эффективность (уровень локального контроля) в отношении метастазов радиочувствительных и радиорезистентных опухолей [14]. Важно отметить, что, несмотря на вариабельность биологической чувствительности к ионизирующему излучению, опухоли ЖКТ относят 
к радиорезистентным, таким образом, проведение общего облучения головного мозга является малоперспективным $[1,15]$.

Клинические исследования прошлых лет показали, что применение радиохирургии для лечения метастазов опухолей ЖКТ в головном мозге обеспечивает значительно более высокий уровень локального контроля и более длительную продолжительность жизни пациентов по сравнению с облучением всего объема головного мозга $[12,16,17]$.

B paботе Jung et al. [18] проведен сравнительный анализ различных тактик лечения метастазов рака толстого кишечника и прямой кишки в головной мозг. Авторы определили, что медиана продолжительности жизни после выявления метастазов в головном мозге составила 1,5 месяца для пациентов, получавших только стероидную терапию; 4,5 месяца для пациентов, прошедших курс облучения всего объема головного мозга; 9,5 месяцев для пациентов, которым было проведено радиохирургическое лечение, и 11,5 месяцев для тех, кому выполнялось нейрохирургическое удаление. Последнюю группу составили пациенты с одиночными очагами. По данным разных авторов, медиана продолжительности жизни у пациентов с метастазами рака ЖКТ в головном мозге после радиохирургии варьирует от 5,1 до 9,5 месяцев [17-22]. В настоящем исследовании медиана выживаемости пациентов после радиохирургического лечения на аппарате Гамма-Нож составила 8 месяцев. Продолжительность жизни пациентов различалась в 2 и более раза в зависимости от прогностического класса по системе RPA: 4,5 месяца для пациентов, относящихся к наименее благоприятному классу RPA III, 9,7 месяцев для пациентов промежуточного класса RPA II и 18,5 месяца для пациентов наиболее благоприятного класса RPA I. Значимыми факторами прогноза после лечения оказались возраст, состояние по шкале Карновского, наличие экстракраниальных метастазов и количество метастазов в головном мозге. Одиночный метастатический очаг в головном мозге и контроль системного заболевания подтверждаются как независимые благоприятные прогностические параметры в других публикациях [21, 23].

По результатам данного исследования, локальный контроль (стабилизация или уменьшение облученных метастатических очагов) после радиохирургического лечения на аппарате Гамма-Нож был достигнут у 94\% пациентов. Высокая эффективность радиохирургического лечения метастазов рака ЖКТ в головном мозге подтверждается другими авторами: Hasegawa et al. [19] и Matsunaga et al. [24] определили уровень локального контроля метастазов рака ЖКТ в головном мозге после радиохирургического лечения на Гамма-Ноже в $84 \%$ и 91\%, соответственно. Kosaki et al. [25], Skeie et al. [26] и Trifiletti et al. [22] отметили зависимость локального контроля от размера метастатического очага и предписанной дозы облучения: лучший результат был достигнут при радиохирургии метастазов небольшого размера (объем опухоли менее $5 \mathrm{~cm}^{3}$ ) и применении более высоких доз облучения. В нашем исследовании подобная закономерность не определялась вследствие малого числа зафиксированных случаев продолженного роста опухоли после радиохирургического лечения (всего 4 случая). Однако следует отметить, что на продолжительность жизни и время до появления новых метастазов в головном мозге данные параметры статистически значимо не влияли.

Появление новых очагов за период наблюдения было отмечено у 47\% пациентов. Экстракраниальное прогрессирование и возникновение множественных очагов в головном мозге происходило одновременном, что указывало на особенность метастазирования рака ЖКТ, имеющего склонность к стремительной генерализации.

\section{Заключение}

Поражение головного мозга при раке ЖКТ встречается довольно редко. Вместе с тем наличие метастазов в головном мозге свидетельствует об отсутствии контроля над опухолевым процессом, так как обычно сочетается с множественным поражением внутренних органов. Без целенаправленного лечения продолжительность жизни ограничена одним-двумя месяцами. Современные методы высокодозного стереотаксического облучения в режиме радиохирургии позволяют достичь эффективного локального контроля метастазов в головном мозге и существенно продлить жизнь пациентам, особенно в прогностически благоприятных классах.

\section{Список литературы}

1. Cascino T.L., Leavengood J.M., Kemeny N., Posner J.B. Brain metastases from colon cancer // J. Neurooncol. 1983. - Vol. 1(3). - P. 203-209.

2. WeinbergJ.S., Suki D., Hanbali F., Cohen Z.R., Lenzi R., Sawaya R. Metastasis of esophageal carcinoma to the brain // Cancer. - 2003. - Vol. 98(9). - P. 1925-1933.

3. Gbidini M., Petrelli F., Hahne J.C., et al. Clinical outcome and molecular characterization of brain metastases from esophageal and gastric cancer: a systematic review // Med. Oncol. - 2017. - Vol. 34(4). - P. 62.

4. Nieder C., Pawinski A., Balteskard L. Colorectal cancer metastatic to the brain: time trends in presentation and outcome // Oncology. - 2009. - Vol. 76(5). - P. 369-374.

5. Go P.H., Klaassen Z., Meadows M.C., Chamberlain R.S. Gastrointestinal cancer and brain metastasis: a rare and ominous sign // Cancer. - 2011. - Vol. 117(16). - P. 3630-3640. 
6. Голанов А.В., Банов С.М., Ветлова Е.Р. Метастатическое поражение головного мозга: изменение парадигмы лучевого лечения // Вопросы онкологии. - 2015. - № 61(4). - С. 530-545.

7. Tsao M.N., Rades D., Wirth A., et al. Radiotherapeutic and surgical management for newly diagnosed brain metastasis (es): An American Society for Radiation Oncology evidence-based guideline // Pract. Radiat. Oncol. 2012. - Vol. - 2(3). - P. 210-225.

8. Иванов П.И., Зубаткина И.С., Тумарова Д.И. и др. Анализ результатов радиохирургического лечения на аппарате Гамма-Нож (Leksell Gamma Knife) пациентов с метастазами меланомы в головной мозг // Вопросы онкологии. - 2015. - №61(4). - С. 586-591.

9. Никитин Д.И., Зубаткина И.С., Иванов П.И. Радиохирургическое лечение метастазов почечно-клеточного рака на аппарате «Гамма-Нож» // РМЖ. - 2017. - № 16. - С. 1164-1168.

10. Gaspar L., Scott C., Rotman M., et al. Recursive partitioning analysis (RPA) of prognostic factors in three Radiation Therapy Oncology Group (RTOG) brain metastases trials // Int. J. Radiant. Oncol. Biol. Phys. - 1997. Vol. 37(4). - P. 745-751.

11. Michl M., ThurmaierJ., Schubert-Fritschle G., et al. Brain Metastasis in Colorectal Cancer Patients: Survival and Analysis of Prognostic Factors // Clin. Colorectal. Cancer. - 2015. - Vol. 14(4). - P. 281-290.

12. Nozawa H., Ishihara S., Kawai K., et al. Brain Metastasis from Colorectal Cancer: Predictors and Treatment Outcomes // Oncology. - 2017.

13. Sundermeyer M.L., Meropol N.J., Rogatko A., et al. Changing patterns of bone and brain metastases in patients with colorectal cancer // Clin. Colorectal. Cancer. - 2005. - Vol. 5(2). - P. 108-113.

14. Yaeb A., Nanda T., Jani A., et al. Control of brain metastases from radioresistant tumors treated by stereotactic radiosurgery // J. Neurooncol. - 2015. - Vol. 124(3). - P. 507-514.

15. Deschavanne P.J., Fertil B. A review of human cell radiosensitivity in vitro // Int. J. Radiat. Oncol. Biol. Phys. 1996. - Vol. 34(1). - P. 251-266.

16. Mege D., Sans A., Ouaissi M., et al. Brain metastases from colorectal cancer: characteristics and management // ANZ J. Surg. - 2017.

17. Park Y.S., ChangJ.H., ChangJ.W., Park Y.G. The efficacy of gamma knife radiosurgery for advanced gastric cancer with brain metastases // J. Neurooncol. - 2011. - Vol. 103(3). - P. 513-521.

18.Jung M., Ahn J.B., ChangJ.H., et al. Brain metastases from colorectal carcinoma: prognostic factors and outcome // J. Neurooncol. - 2011. - Vol. 101(1). - P. 49-55.

19. Hasegawa T., Kondziolka D., Flickinger J.C., Lunsford L.D. Stereotactic radiosurgery for brain metastases from gastrointestinal tract cancer // Surg. Neurol. - 2003. - Vol. 60(6). - P. 506-514.

20. Page B.R., Wang E.C., White L., et al. Gamma Knife radiosurgery for brain metastases from gastrointestinal primary // J. Med. Imaging Radiat. Oncol. - 2017. - Vol. 61(4). - P. 522-527.

21. Paix A., Antoni D., Adeduntan R., Noël G. Stereotactic radiation therapy of brain metastases from colorectal cancer: A single institution cohort // Cancer Radiother. - 2017. - Vol. 21(3). - P. 199-204.

22. Trifiletti D.M., Patel N., Lee C.C., et al. Stereotactic radiosurgery in the treatment of brain metastases from gastrointestinal primaries // J. Neurooncol. - 2015. - Vol. 124(3). - P. 439-446.

23. Gu X.D., Cai Y.T., Zhou Y.M., et al. Prognostic factors and multidisciplinary treatment modalities for brain metastases from colorectal cancer: analysis of 93 patients // BMC Cancer. - 2015. - Vol. 15. - P. 902.

24. Matsunaga S., Shuto T., Kawahara N. et al. Gamma Knife surgery for brain metastases from colorectal cancer // J. Neurosurg. - 2011. - Vol. 114(3). - P. 782-789.

25. Kosaki K., Shibamoto Y., Hirai T., et al. Regression curves of brain metastases after gamma knife irradiation: Difference by tumor and patient characteristics // Cancer Sci. - 2012. - Vol. 103(11). - P. 1967-1973.

26. Skeie B.S., Enger P.Ø., Ganz J.C., et al. Gamma knife surgery of colorectal brain metastases: a high prescription dose of 25 Gy may improve growth control // World Neurosurg. - 2013. - Vol. 79(3-4). - P. 525-536.

\section{References}

1. Cascino T.L., Leavengood J.M., Kemeny N., Posner J.B. Brain metastases from colon cancer. J. Neurooncol. 1983; 1(3): 203-209. PMID: 6678969.

2. WeinbergJ.S., Suki D., Hanbali F., Cohen Z.R., Lenzi R., Sawaya R. Metastasis of esophageal carcinoma to the brain. Cancer. 2003; 98(9): 1925-1933. doi: 10.1002/cncr.11737. PMID: 14584076.

3. Ghidini M., Petrelli F., Habne J.C., De Giorgi A., Toppo L., Pizzo C., Ratti M., Barni S., Passalacqua R., Tomasello G. Clinical outcome and molecular characterization of brain metastases from esophageal and gastric cancer: a systematic review. Med.Oncol. 2017; 34(4): 62. doi: 10.1007/s12032-017-0919-0. PMID: 28315230.

4. Nieder C., Pawinski A., Balteskard L. Colorectal cancer metastatic to the brain: time trends in presentation and outcome. Oncology. 2009; 76(5): 369-374. doi: 10.1159/000210026. PMID: 19321946.

5. Go P.H., Klaassen Z., Meadows M.C., Chamberlain R.S. Gastrointestinal cancer and brain metastasis: a rare and ominous sign. Cancer. 2011; 117(16): 3630-3640. doi: 10.1002/cncr.25940. PMID: 21319152.

6. [Golanov A.V., Banov S.M., Vetlova E.R. Metastatic brain damage: a paradigm shift in radiation treatment. Questions of Oncology. 2015; 61(4): 530-545. PMID: 26571820].

7. Tsao M.N., Rades D., Wirth A., Lo S.S., Danielson B.L., Gaspar L.E., Sperduto P.W., Vogelbaum M.A., Radawski J.D., Wang J.Z., Gillin M.T., Mobideen N., Habn C.A., Chang E.L. Radiotherapeutic and surgical management for newly 
diagnosed brain metastasis (es): An American Society for Radiation Oncology evidence-based guideline. Pract. Radiat. Oncol. 2012; 2(3): 210-225. doi: 10.1016/j.prro.2011. PMID: 25925626.

8. [Ivanov P.I., Zubatkina I.S., Tumarova D.I., Andreev G.I., Volkov N.M. Analysis of the results of radiosurgical treatment on the Gamma-Knife apparatus (Leksell Gamma Knife) of patients with metastasis of melanoma in the brain. Questions of Oncology. 2015; 61(4): 586-591. PMID: 26571828].

9. [Nikitin D.I., Zubatkina I.S., Ivanov P.I. Radiosurgical treatment of metastases of renal cell carcinoma on the apparatus «Gamma-Knife». RMZH. 2017; 16: 1164-1168].

10. Gaspar L., Scott C., Rotman M., Asbell S., Phillips T., Wasserman T., McKenna W.G., Bybardt R. Recursive partitioning analysis (RPA) of prognostic factors in three Radiation Therapy Oncology Group (RTOG) brain metastases trials. Int. J. Radiant. Oncol. Biol. Phys. 1997; 37(4): 745-751. PMID: 9128946.

11. Michl M., Thurmaier J., Schubert-Fritschle G., Wiedemann M., Laubender R.P., Nüssler N.C., Ruppert R., KleeffJ., Schepp W., Reuter C., Löhe F., Karthaus M., NeumannJ., Kirchner T., EngelJ., Heinemann V. Brain Metastasis in Colorectal Cancer Patients: Survival and Analysis of Prognostic Factors. Clin. Colorectal. Cancer. 2015; 14(4): 281-290. doi: 10.1016/j.clcc.2015.05.009. PMID: 26123495.

12. Nozawa H., Ishihara S., Kawai K., Sasaki K., Murono K., Otani K., Nishikawa T., Tanaka T., Kiyomatsu T., Hata K., Watanabe T. Brain Metastasis from Colorectal Cancer: Predictors and Treatment Outcomes. Oncology. 2017. doi: 10.1159/000478661. PMID: 28700994.

13. Sundermeyer M.L., Meropol N.J., Rogatko A., Wang H., Cohen S.J. Changing patterns of bone and brain metastases in patients with colorectal cancer. Clin. Colorectal. Cancer. 2005; 5(2): 108-113. PMID: 16098251.

14. Yaeb A., Nanda T., Jani A., Rozenblat T., Qureshi Y., Saad S., Lesser J., Lassman A.B., Isaacson S.R., Sisti M.B., Bruce J.N., McKhann G.M. 2nd, Wang T.J. Control of brain metastases from radioresistant tumors treated by stereotactic radiosurgery. J. Neurooncol. 2015; 124(3): 507-514. doi: 10.1007/s11060-015-1871-5. PMID: 26233247.

15. Deschavanne P.J., Fertil B. A review of human cell radiosensitivity in vitro. Int. J. Radiat. Oncol. Biol. Phys. 1996; 34(1): 251-266. PMID: 12118559.

16. Mege D., Sans A., Ouaissi M., Iannelli A., SielezneffI. Brain metastases from colorectal cancer: characteristics and management. ANZ J. Surg. 2017. doi: 10.1111/ans.14107. PMID: 28687024.

17.Park Y.S., ChangJ.H., ChangJ.W., Park Y.G. The efficacy of gamma knife radiosurgery for advanced gastric cancer with brain metastases. J. Neurooncol. 2011; 103(3): 513-521. doi: 10.1007/s11060-010-0405-4. PMID: 20957408.

18. Jung M., Ahn J.B., Chang J.H., Sub C.O., Hong S., Roh J.K., Shin S.J., Rha S.Y. Brain metastases from colorectal carcinoma: prognostic factors and outcome. J. Neurooncol. 2011; 101(1): 49-55. doi: 10.1007/s11060-010-0214-9. PMID: 20467783.

19. Hasegawa T., Kondziolka D., Flickinger J.C., Lunsford L.D. Stereotactic radiosurgery for brain metastases from gastrointestinal tract cancer. Surg. Neurol. 2003; 60(6): 506-514. PMID: 14670663.

20. Page B.R., Wang E.C., White L., McTyre E., Peiffer A., Alistar A., Mu F., Loganathan A., Bourland J.D., Laxton A.W., Tatter S.B., Chan M.D. Gamma Knife radiosurgery for brain metastases from gastrointestinal primary. J. Med. Imaging Radiat. Oncol. 2017; 61(4): 522-527. doi: 10.1111/1754-9485.12584. PMID: 28139076.

21. Paix A., Antoni D., Adeduntan R., Noël G. Stereotactic radiation therapy of brain metastases from colorectal cancer: A single institution cohort. Cancer Radiother. 2017; 21(3): 199-204. doi: 10.1016/j.canrad.2017.01.010. PMID: 28499660.

22. Trifiletti D.M., Patel N., Lee C.C., Romano A.M., Sheehan J.P. Stereotactic radiosurgery in the treatment of brain metastases from gastrointestinal primaries. J. Neurooncol. 2015; 124(3): 439-446. doi: 10.1007/s11060-015-1857-3. PMID: 26186901.

23. Gu X.D., Cai Y.T., Zhou Y.M., Li Z.Y., Xiang J.B., Chen Z.Y. Prognostic factors and multidisciplinary treatment modalities for brain metastases from colorectal cancer: analysis of 93 patients. BMC Cancer. 2015; 15: 902. doi: 10.1186/s12885-015-1933-2. PMID: 26572484.

24. Matsunaga S., Shuto T., Kawahara N., Suenaga J., Inomori S., Fujino H. Gamma Knife surgery for brain metastases from colorectal cancer. J. Neurosurg. 2011; 114(3): 782-789. doi: 10.3171/2010.9.JNS10354. PMID: 20950083.

25. Kosaki K., Shibamoto Y., Hirai T., Hatano M., Tomita N., Kobayashi T., Mori Y. Regression curves of brain metastases after gamma knife irradiation: Difference by tumor and patient characteristics. Cancer Sci. 2012; 103(11): 1967-1973. doi: 10.1111/j.1349-7006.2012.02392.x. PMID: 22988917.

26. Skeie B.S., Enger P.Ø., Ganz J.C., Skeie G.O., Parr E., Hatteland S., Ystevik B., Heggdal J.I., Pedersen P.H. Gamma knife surgery of colorectal brain metastases: a high prescription dose of $25 \mathrm{~Gy}$ may improve growth control. World Neurosurg. 2013; 79(3-4): 525-536. doi: 10.1016/j.wneu.2011.09.019. PMID: 22120263. 